\title{
Correction: Molecular Characterization of Transcriptome-wide Interac- tions between Highly Pathogenic Porcine Reproductive and Respiratory Syndrome Virus and Porcine Alveolar Macrophages in vivo
}

\section{Ping Zhou, Shanli Zhai, Xiang Zhou, Ping Lin, Tengfei Jiang, Xueying Hu, Yunbo Jiang, Bin Wu, Qingde Zhang, Xuewen Xu, Jin-ping Li, Bang Liu ${ }^{\bowtie}$}

$\triangle$ Corresponding author: liubang@mail.hzau.edu.cn; Tel: +86 27 87284140; Fax: +86 2787280408

Published: 2011.12.01

Corrected article: Int J Biol Sci 20 I ; 7(7):947-959.

During the revision process several genes for Q-PCR validation were changed in the Figure 4 according to the suggestion from the reviewers, however, the Q-PCR primers information in the Table 1 has been ignored to be corrected accordingly. Here, the corrected version of the Table 1 is shown below. We apologize for this oversight and for any confusion that it has caused.

Table I Primers used for Q-PCR validation.

\begin{tabular}{|c|c|c|c|}
\hline Gene & Primer sequence $\left(5^{\prime}-3^{\prime}\right)$ & Target size (bp) & $\operatorname{Tm}\left({ }^{\circ} \mathrm{C}\right)^{\mathrm{a}}$ \\
\hline ATP6V1B2 & $\begin{array}{l}\text { Forward: CAAGCCATGAAAGCCGTAGTT } \\
\text { Reverse: TGCCAGCCAATGTCCAAAGT }\end{array}$ & 149 & 60 \\
\hline C3 & $\begin{array}{l}\text { Forward: AAACTAAAGGAGGGGGGACACT } \\
\text { Reverse: CTTGGCATACATCACCATCAGG }\end{array}$ & 133 & 60 \\
\hline CCL2 & $\begin{array}{l}\text { Forward: AACTTGCCCTAAATACCCTCAGA } \\
\text { Reverse: GGAAAGCAATGTGCCCAAGTC }\end{array}$ & 179 & 61 \\
\hline DDIT3 & $\begin{array}{l}\text { Forward: ACGGCTCAAGCAGGAAATC } \\
\text { Reverse: CACTGGTAAGAAGGTGGTTGGT }\end{array}$ & 173 & 58 \\
\hline GLRX2 & $\begin{array}{l}\text { Forward: TACGGAAGCCAGTTTCAAGAC } \\
\text { Reverse: CTTGGTGAAGCCTATGAGTGTC }\end{array}$ & 118 & 58 \\
\hline SLC39A14 & $\begin{array}{l}\text { Forward: TCTCTGCCTGCTGCTGTTACG } \\
\text { Reverse: GCCTTCCTTTCATCCTCTTGG }\end{array}$ & 166 & 60 \\
\hline TNF & $\begin{array}{l}\text { Forward: CATCGCCGTCTCCTACCA } \\
\text { Reverse: CCCAGATTCAGCAAAGTCCA }\end{array}$ & 199 & 58 \\
\hline RPL32b & $\begin{array}{l}\text { Forward: CGGAAGTTTCTGGTACACAATGTAA } \\
\text { Reverse: TGGAAGAGACGTTGTGAGCAA }\end{array}$ & 94 & $58-61$ \\
\hline
\end{tabular}

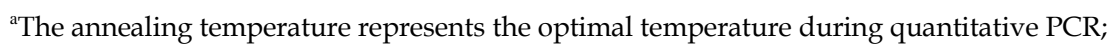

${ }^{\mathrm{b}} \mathrm{RNA}$ levels of RPL32 was assayed for normalization during quantitative PCR. 